\title{
Author Correction: Atg9 is a lipid scramblase that mediates autophagosomal membrane expansion
}

Kazuaki Matoba DD, Tetsuya Kotani, Akihisa Tsutsumi (D), Takuma Tsuji, Takaharu Mori, Daisuke Noshiro, Yuji Sugita (D), Norimichi Nomura (D), So Iwata (D), Yoshinori Ohsumi, Toyoshi Fujimoto (D), Hitoshi Nakatogawa (D), Masahide Kikkawa (D) and Nobuo N. Noda (D)

Correction to: Nature Structural \& Molecular Biology https://doi.org/10.1038/s41594-020-00518-w, published online 26 October 2020.

In the version of this article initially published online, the reference "Guardia, C. M. et al. Structure of human ATG9A, the only transmembrane protein of the core autophagy machinery. Cell Rep. 31, 107837 (2020)" was omitted. It should have been cited after "A very recent report..." and after "PDB 6WQZ" in the caption for Extended Data Fig. 7a. The error has been corrected in the print, PDF and HTML versions of the article.

Published online: 12 November 2020

https://doi.org/10.1038/s41594-020-00538-6

(c) The Author(s), under exclusive licence to Springer Nature America, Inc. 2020

\section{Author Correction: Quantifying the dynamics of IRES and cap translation with single-molecule resolution in live cells}

\author{
Amanda Koch, Luis Aguilera, Tatsuya Morisaki, Brian Munsky (D) and Timothy J. Stasevich (D)
}

Correction to: Nature Structural \& Molecular Biology https://doi.org/10.1038/s41594-020-0504-7, published online 21 September 2020.

In the version of this article initially published online, there were errors in some of the figures and figure captions. In Extended Data Fig. 2, panels were missing. The correct version of the figure, which contains four additional panels (a-d), is shown below.

In Fig. 5a, the heading "Promoter states" in the box on the left was incorrect. The correct heading is "mRNA states." In the Fig. 5a caption, it incorrectly stated "The selected model considers four promoter states...;" the correct wording is "The selected model considers four mRNA states." In the footnote in Table 1, it incorrectly stated "Four promoter states were proposed...;" the correct wording is "Four mRNA states were proposed." Also in the Fig. 5a caption, the callout to Supplementary Fig. 6c,d was incorrect; the correct callout is to Extended Data Fig 6c,d.

In the caption for Extended Data Fig. 6c, it incorrectly stated "The first mechanism mimics the inhibition of the Cap activation rates at the promoter level...;" the correct wording is "The first mechanism mimics the inhibition of the Cap activation rates at the mRNA level...."

In the Extended Data Fig. 4b legend, the callout to Fig. 4 was incorrect; the correct callout is to Fig. 3. The errors have been corrected in the print, PDF and HTML versions of the article. 

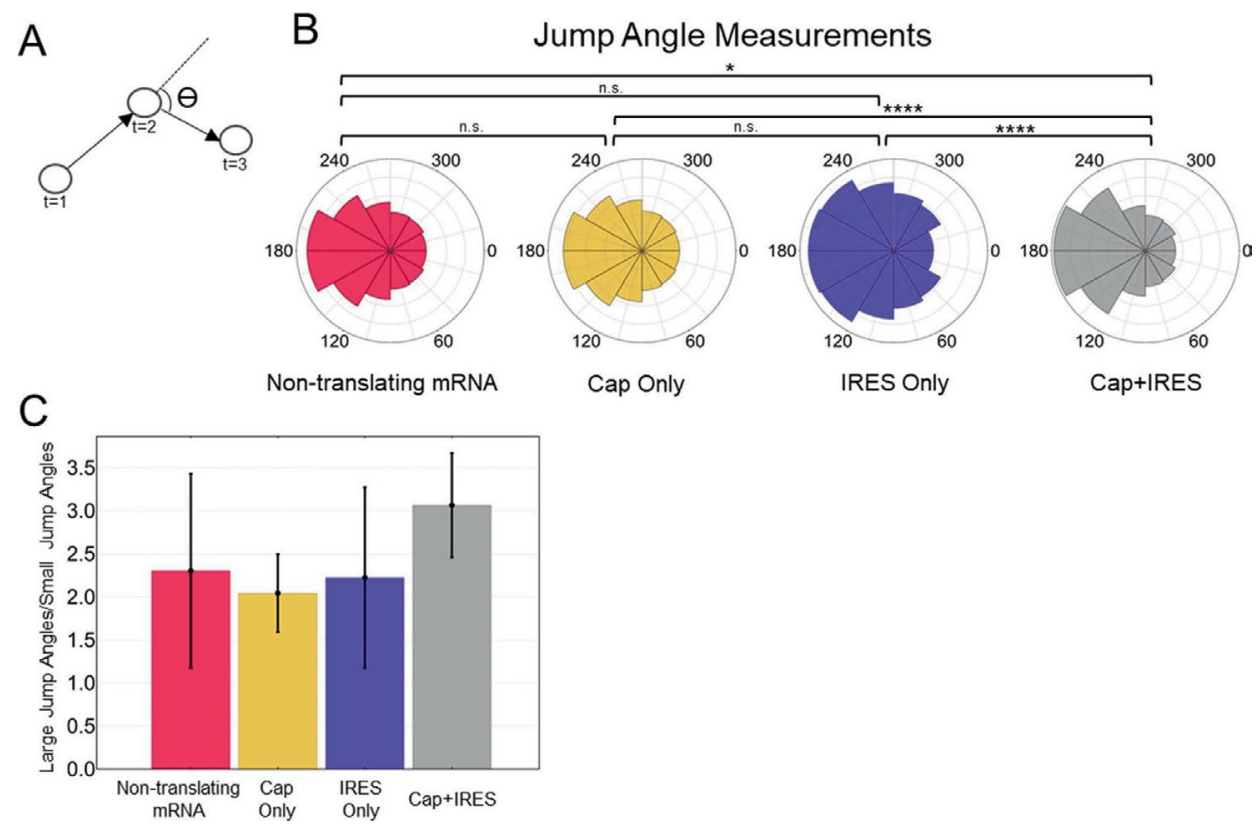

Corrected

A

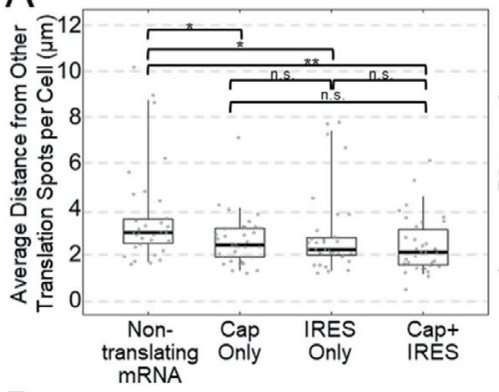

$\mathrm{D}$

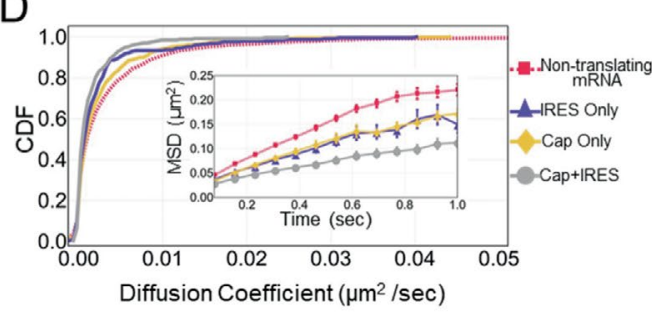

B

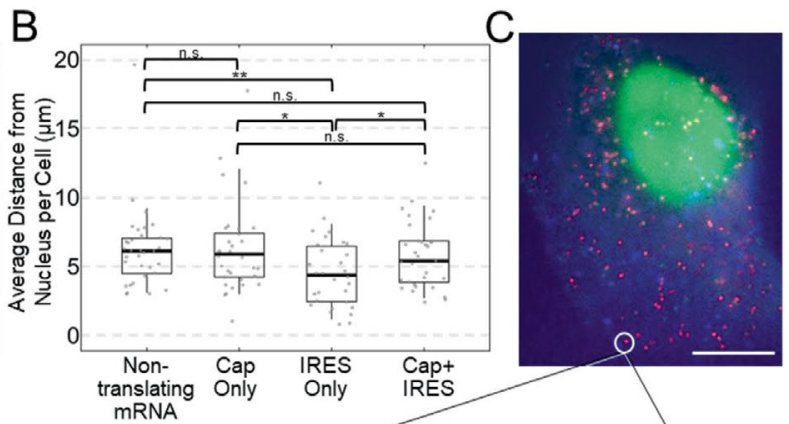

E

$\mathrm{F}$
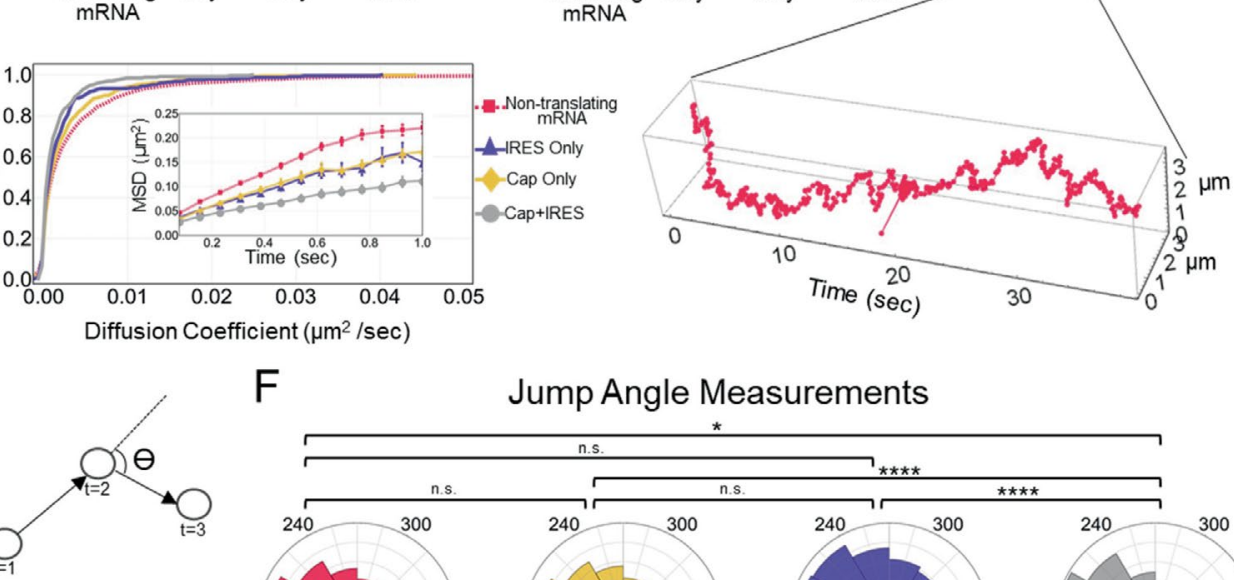

180

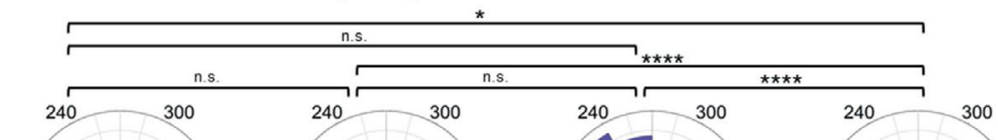

180

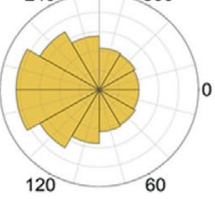

Cap Only

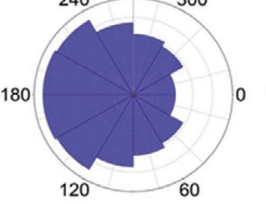

IRES Only

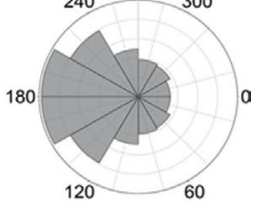

Cap+IRES

Extended Data Fig. 2 | Original and Corrected.

Published online: 27 October 2020

https://doi.org/10.1038/s41594-020-00531-z

(C) The Author(s), under exclusive licence to Springer Nature America, Inc. 2020 